\title{
The application of accelerometers to measure movements of upper limbs: Pilot study
}

\author{
Patrik Kutilek $^{1,2}$, Petr Volf ${ }^{1, *}$, Rudolf Cerny ${ }^{3}$, and Jan Hejda ${ }^{1}$ \\ ${ }^{I}$ Faculty of Biomedical Engineering, Czech Technical University in Prague, Kladno, Czech Republic; ${ }^{2}$ General Univer- \\ sity Hospital - $I^{\text {st }}$ Faculty of Medicine, Charles University in Prague, Prague, Czech Republic; and ${ }^{3}$ University Hospital \\ Motol - $2^{\text {nd }}$ Faculty of Medicine, Charles University in Prague, Prague, Czech Republic
}

Copyright: (C) 2017 P. Kutilek et al. This is an open access article licensed under the Creative Commons Attribution License (http://creativecommons.org/licenses/by/4.0/).

\begin{abstract}
Background: Even though inertial measurement units (IMU) are already being used experimentally for evaluating movements of segment of the axial skeleton, no studies have been found which have used IMUs to measure the behavior of the segments of upper limbs during quiet stance. Objective: The objective is to design a suitable application of IMUs to measure movements of the upper extremities in Romberg's test and analyze spontaneous arm movements. Second aim is to identify possible discrepancies between the dominant and non-dominant arm movements. Methods: The dominant and non-dominant upper limb of each participant was identified. Then, the movements of both upper limbs were measured by the Xsens system equipped with MTx motion trackers during the quiet stance on a firm surface with eyes open (EO) and eyes closed (EC). The measured data was used to calculate the medians and maximums of the superior-inferior, medio-lateral and anterior-posterior acceleration. Also, tremor intensity was calculated to quantitatively evaluate the measured data. Results: The comparison of values of maximal accelerations of the dominant and non-dominant arms showed significant difference between the arms during EC conditions. The comparison of values of median accelerations of the dominant and non-dominant arms showed significant differences between the acceleration of arms in medio-lateral direction during EO and EC conditions. In all cases, values of maximal and median accelerations and values of tremor intensity of the dominant limb strongly correlated with values on the non-dominant limb. Conclusions: Findings suggest possible usefulness of the designed application of IMUs and evaluation methods for their use in Romberg's test in clinical practice for evaluation of upper limb movements.
\end{abstract}

Keywords: acceleration, upper limb, tremor intensity, quiet stance, Romberg's test, handedness

\section{Introduction}

Movements of body segments during stance tasks can indicate the condition of the musculoskeletal or nervous system (e.g., Duclos, Nadeau, \& Lecours, 2008). Romberg's test has become standard in the evaluation of spontaneous movements during quiet standing (Khasnis \& Gokula, 2003). Recently, also less expensive inertial measurement units (IMU), which represent electronic devices based on the accelerometers and gyroscopes, were used for high-accuracy measurement of human body segments during quiet stance, instead of commonly used posturography platforms (Mancini et al., 2012). In the past, sensing units were placed on segments of the axial skeleton (i.e. trunk

\footnotetext{
* Address for correspondence: Petr Volf, Department of Biomedical Technology, Faculty of Biomedical Engineering, Czech Technical University in Prague, nám. Sítná 3105, 27201 Kladno, Czech Republic. E-mail: petr.volf4@fbmi.cvut.cz
}

or head) for measuring the motion of these segments during quite stance (Liu, Zhang, \& Lockhart, 2012). Even though IMUs are already being used experimentally for measuring movements of the head and trunk, no studies have been found which would use IMUs to measure and evaluate the behavior of the segments of the appendicular skeleton during quiet stance. Spontaneous movements of the extremity in rest or during postural activity called physiological tremor (PT) can be measured by IMUs. It is apparent, that measuring segments of the appendicular skeleton may contribute to the indication of condition of musculoskeletal or nervous system. Physiological tremor is not apparent to the naked eye and does not interfere with the activities of daily life. On the other hand, enhanced physiological tremor can disturb voluntary movements and has to be distinguished from essential, metabolic tremor or tremor due to the Parkinson's disease. Clinical and 
biomechanical characteristics of different tremor types are important for correct diagnose (Louis, 2005).

The first objective of this work is to design a suitable application of inexpensive accelerometers to measure movements of the upper extremities during stance tasks, design quantitative methods of data analysis and test their application on healthy subjects.

The second aim of the study is, by measuring healthy subjects, to identify discrepancies between the movements of dominant and non-dominant upper arm in anatomical axes. Thus, the research question is whether there are also measurable tremors in healthy population, and if tremors are different for the dominant and non-dominant upper limb.

\section{Methods}

\section{Test procedure and participants}

The set of data to test the applicability of the IMUs for measuring human arm motion during quiet stance was measured on the sample of fourteen volunteers aged 22 ( $S D$ 0.5) years; body weight 71 ( $S D$ 12) kg; height $176(S D 8) \mathrm{cm}$ were recruited from students (healthy subjects) of the Czech Technical University in Prague. The Edinburgh Handedness Inventory was used to assess the dominance of a person's right or left upper limb (e.g. Oldfield, 1971). In the study, there was one weak left-hander (handedness 9\%) and thirteen right-handers (from weak [11\%] to strong handedness [100\%]) among the subjects. The mean handedness score of the group was $49 \%$ (SD 31\%). The diagnostic evaluation included a detailed disease history, and an informed consent was obtained from each subject. The study was performed in accordance with the Helsinki Declaration. The study protocol was approved by the local Ethics Committee of the Faculty of Biomedical Engineering of the Czech Technical University (CTU) in Prague. The subjects were chosen for measurement randomly, and on different days.

\section{Test procedure and measurement equipment}

Xbus Master (Xsens Technologies B.V., Enschede, Netherlands), a lightweight (330 g) and portable device using MTx units for orientation and acceleration measurement of body segments (see Figure 1), was used for measurement of dominant and nondominant upper arm movements. An MTx unit with an embedded accelerometer and gyroscope represents an accurate IMU measuring drift-free 3-D orientation and 3-D acceleration (Kutílek, Socha, Čakrt, Schlenker, \& Bizovská, 2015). The MTx unit was set up in a way that one axis of the coordinate system of the MTx unit was parallel to the anterior-posterior axis, i.e. symmetry axis of the fixed stationary platform on which the participants stood, and the other two axes were perpendicular to the anterior-posterior axis (i.e. symmetry axis of the platform) with respect to the direction of the Earth's gravity, i.e. superior-inferior axis was co-linear with the direction of gravity. IMU collected acceleration data at a sampling rate of $50 \mathrm{~Hz}$. Bias stability of the accelerometer is $0.02 \mathrm{~m} \cdot \mathrm{s}^{-2}$, full scale (standard) is $\pm 17 \mathrm{~m} \cdot \mathrm{s}^{-2}$, linearity is $0.05 \%$ of full scale, scale factor stability is $0.05 \%$ and noise density is $0.001 \mathrm{~m} \cdot \mathrm{s}^{-2} \cdot \mathrm{Hz}^{-0.5}$. Orientation performance of gyroscopes is: angular resolution $0.05^{\circ}$, static accuracy $<0.5^{\circ}$, dynamic accuracy $2^{\circ}$.

After the adjustment, MTx units were placed on the subject's upper extremities in compliance with studies by Rodríguez et al. (2010) and Stirling, Hesami, Ritz, Adistambha, and Naghdy (2010), and in accordance with the MoCap system manufacturer recommendation. Two MTx units were placed on dorsal sides of upper arms close to centers of mass of segments without depriving motion of upper extremities or trunk.

The data, i.e. the three Euler angles (roll [Ф], yaw $[\Psi]$, pitch $[\Theta]$ ) (Allum, Nijhuis, \& Carpenter, 2008; Aw, Halmagyi, Black, Curthoys, Yavor, \& Todd, 1998; Findling et al., 2011) and three orthogonal accelerations $\left(a_{S x}, a_{S y}, a_{S z}\right)$ in the accelerometer coordinate system (Kennie \& Petrie, 2010) were measured by a MTx unit placed on the upper arms while subjects were performing a quiet stance on a fixed stationary platform. Conventions of Euler angles are described in studies by Allum et al. (2008), Findling et al. (2011) and Osler and Reynolds (2012). The three accelerations measured by the accelerometer of MTx unit are described

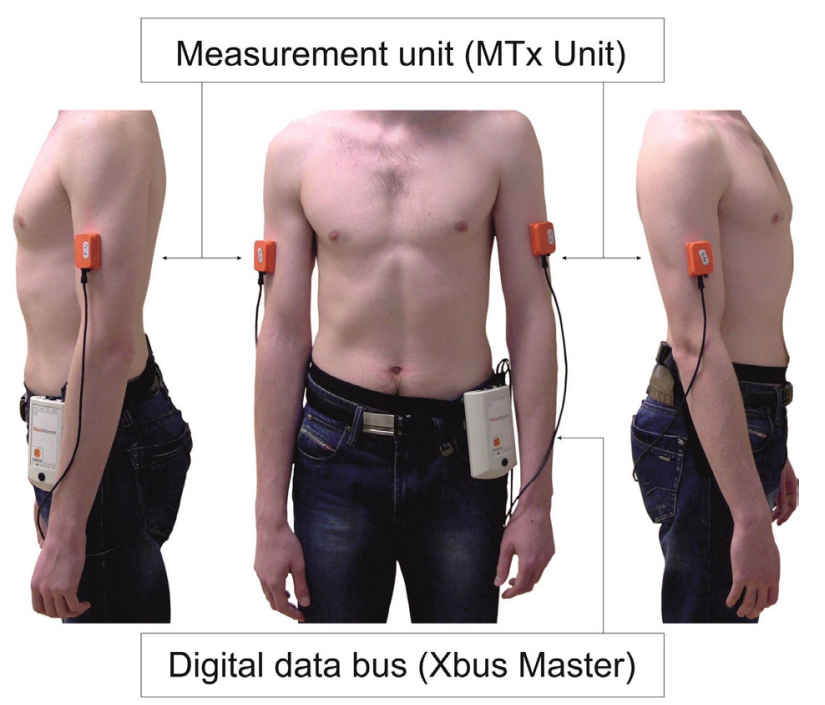

Figure 1. Arrangement of the Xsens system with two three-axis IMUs used to measure acceleration of upper limbs. 
in detail by Altun and Barshan (2012) and Gil-Agudo et al. (2013).

The movements of both upper arms (dominant and non-dominant) were measured by the Xsens system during the quiet stance (i.e. Romberg's test) on a firm surface (FiS) with eyes open (EO) and eyes closed (EC) (e.g., Honegger, Van Spijker, \& Allum, 2012). The subject's bare feet were positioned next to each other, splayed at an angle of $30^{\circ}$, arms always in a hanging position. The tasks included standing on both feet for at least 60 seconds (Zadnikar \& Rugelj, 2011).

\section{Method of data processing}

Three orthogonal accelerations and three Euler angles were recorded continuously during trials. This way, a set of instantaneous values were obtained by both MTx units placed on upper arms. The instantaneous values of the three Euler angles and three accelerations in the accelerometer coordinate system of each MTx unit were used to calculate the accelerations in the global reference system and then in the anatomical coordinate frame. The calculation is based on the rotational matrices. The first rotation matrix $R_{G S i}$ rotates an acceleration vector $\boldsymbol{a}_{S i}=\left(a_{S x i} a_{S y i} a_{S z i}\right)^{T}$ of instantaneous (i) accelerations in the sensor co-ordinate system (S) to the global (G) reference system $\boldsymbol{a}_{G i}=R_{G S i} \cdot \boldsymbol{a}_{S i}$, where the matrix $R_{G S i}$ is described in detail in (Ying \& Kim, 2002). The acceleration vector $\boldsymbol{a}_{G i}=\left(a_{G x i} a_{G y i} a_{G z i}\right)^{T}$ of the instantaneous accelerations in the global reference system is then rotated to the anatomical (A) coordinate frame $\boldsymbol{a}_{A i}=R_{A G} \cdot \boldsymbol{a}_{G i}$ (Brinckmann, Frobin, \& Leivseth, 2002). The second rotation matrix $R_{A G}$ is obtained during the calibration process of the MTx unit. The calibration method and the application of the rotation matrix are described in detail in Melecky et al. (2016). The calculated acceleration vector $\boldsymbol{a}_{A i}=\left(a_{A P i} a_{M L i} a_{S I i}\right)^{T}$ of the instantaneous accelerations represents the superior-inferior (SI) acceleration $\left(a_{S I i}\right)$, medio-lateral (ML) acceleration $\left(a_{M L i}\right)$ and anterior-posterior (AP) acceleration $\left(a_{A P i}\right)$. Acceleration is recorded continuously during measurements. For quantitative evaluation of the data, the maximal measured values of the accelerations $a_{A P \max }=\max \left(a_{A P l}, \ldots, a_{A P n}\right), a_{M L \max }=\max \left(a_{M L l}, \ldots, a_{M L n}\right)$, $a_{\text {SImax }}=\max \left(a_{S I I}, \ldots, a_{S I n}\right)$ were used in accordance with the study by Schubert, Kirchner, Schmidtbleicher, and Haas (2012). The $n$ is the number of sample points (Kutílek et al., 2015).

Since the mean value and/or median value of the data record is not equal to zero and the signal contains both positive and negative values, the offset correction and rectification of the recorded and calculated accelerations was used in the data record for further evaluation. The baseline of the data record is usually shifted away from the true zero line of recorded accelerations (median value of the acceleration is not zero), therefore offset correction is used. The median value of the recorded acceleration is used to shift the baseline at the zero (i.e. the baseline constantly remains at the zero line). Although the mean value and/or median value are close to zero or are zero because of the high number of sample points, the offset correction is used in data processing. Rectification involved the transformation of the signal into only positive deflections, i.e. the negative peaks were "moved up" to plus (Konrad, 2005), see Figure 2. Then, the processed signal was used to calculate the medians, (cf. Hogg \& Craig, 1995), of the modified values of the superior-inferior acceleration $\left(a_{\text {SImed }}\right)$, medio-lateral acceleration $\left(a_{\text {MLmed }}\right)$ and anterior-posterior acceleration $\left(a_{A \text { Pmed }}\right)$ in accordance with the study by Schubert et al. (2012), as an alternative to the calculation of the mean values of the measured data.

Also, the tremor intensity (TI) of the accelerations was used to quantitatively evaluate the measured data. Tremor intensity is the root mean square of acceleration recorded in the specific band (Papapetropoulos et al., 2010). We used a band of 1 to $15 \mathrm{~Hz}$, same as Machowska-Majchrzak, Pierzchała, Pietraszek, and Łabuz-Roszak (2011) and Machowska-Majchrzak, Pierzchała, Pietraszek, Łabuz-Roszak, and Bartman (2012). Fast Fourier Transform (FFT) implemented in the MatLab software (Version R2010b; Mathworks, Natick, MA, USA) was used to determine the distribution of the tremor in the frequency band. TI is measured in $\mathrm{m} \cdot \mathrm{s}^{-2}$; and larger values are indicative of more intense tremor (Papapetropoulos et al., 2010). A custom-designed MatLab program based on the functions of the MatLab software was used to calculate the $a_{\text {APmax }}, a_{\text {MLmax }}, a_{\text {SImax }}, a_{\text {APmed }}, a_{\text {MLmed }}, a_{\text {SImed }}, T I_{A P}, T I_{M L}$ and $T I_{S I}$.

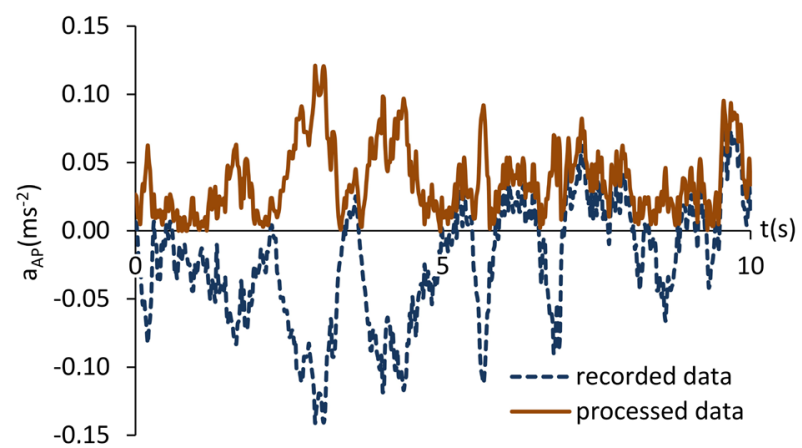

Figure 2. Example of recorded/calculated anteriorposterior acceleration and processed anterior-posterior acceleration. 


\section{Statistical analysis}

After calculating the maximal measured values of accelerations and medians of measured values of accelerations, the data from the two MTx units were divided into two sets; one group included accelerations measured on the dominant upper limb, and the second group included accelerations measured on the nondominant upper limb. Also, the data sets were divided into data subsets according to whether the subjects were standing with EO or EC. The Jarque-Bera test was used to test the normal distribution of calculated median accelerations and maximal accelerations in the data subsets. The median (Med), minimum (Min), maximum (Max), the first quartile (Q1) and the third quartile $(\mathrm{Q} 3)$ of the accelerations were then calculated. The first quartile is defined as the middle number between the smallest number and the median of the data set. The third quartile is the middle value between the median and the highest value of the data set. These indicators are provided because of the statistical presentation of the results and possible future use. Also, the Wilcoxon test was used to assess the significance of the differences between the results of measurements on the dominant and non-dominant arms; and differences between the results of measurements with EO and EC. The significance level was set at .05. In addition, effect sizes (Cohen's $d$ ) (Cohen, 1988), which consider the difference between the two groups of data, were calculated in accordance with studies by Coolican (1990) and Fritz, Morris, and Richler (2012).

Also, the Spearman's rank correlation coefficient between the data subsets were calculated to study the relations between the data measured on the dominant upper limb and the data measured on the non-dominant upper limb; relations between the superior-inferior accelerations, medio-lateral accelerations and anteriorposterior acceleration; and relations between the data measured on subjects with EO and EC. The statistical analysis was performed using MatLab software.

\section{Results}

Jarque-Bera test did not show normal distribution of data in most sets of data. Then, the statistical data were used to illustrate the differences between the movements of the dominant and non-dominant arms of subjects standing with EO and EC. The following plots (Figure 3, Figure 4 and Figure 5) display the Min, Max, Med, Q1, and Q3 for the calculated values of maximum and median accelerations.

\section{Comparing dominant and non-dominant upper limb dur- ing quiet stance trials}

The comparison of values of maximal accelerations of the dominant and non-dominant arm found significant differences between the data measured on subjects with EC, see Table 1. The comparison of values of maximal accelerations of the same arm measured during standing with EO or EC found significant differences only when measuring the non-dominant arm of subjects standing with EC, see Table 1.

The comparison of values of median accelerations of the dominant and non-dominant arm found significant differences between the data for accelerations in ML direction measured on subjects with EO and EC, see Table 1. In almost all cases, the comparison of values of median accelerations of the same arm measured
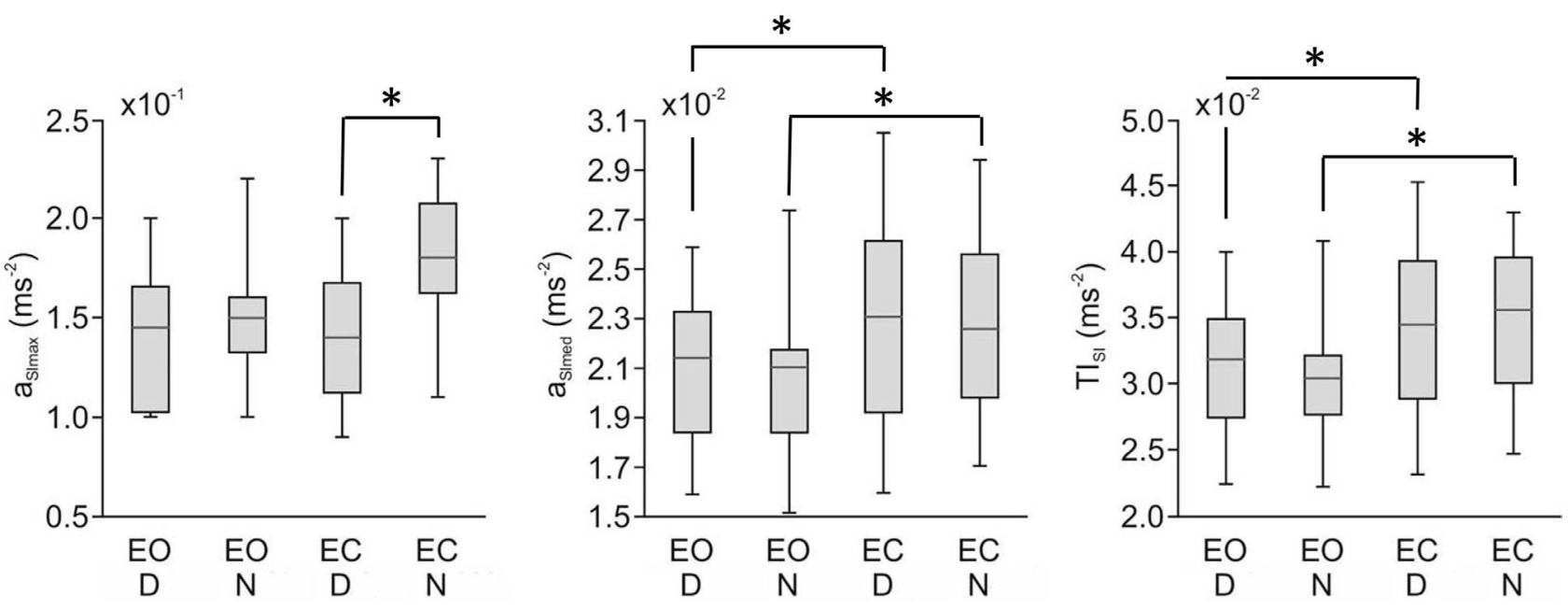

Figure 3. Comparison of maximum (max) and median (med) superior-inferior accelerations and tremor intensity (TI) of superior-inferior acceleration of dominant (D) and non-dominant (N) upper arms of subjects standing with eyes open (EO) and eyes closed (EC). *statistically significant difference. 

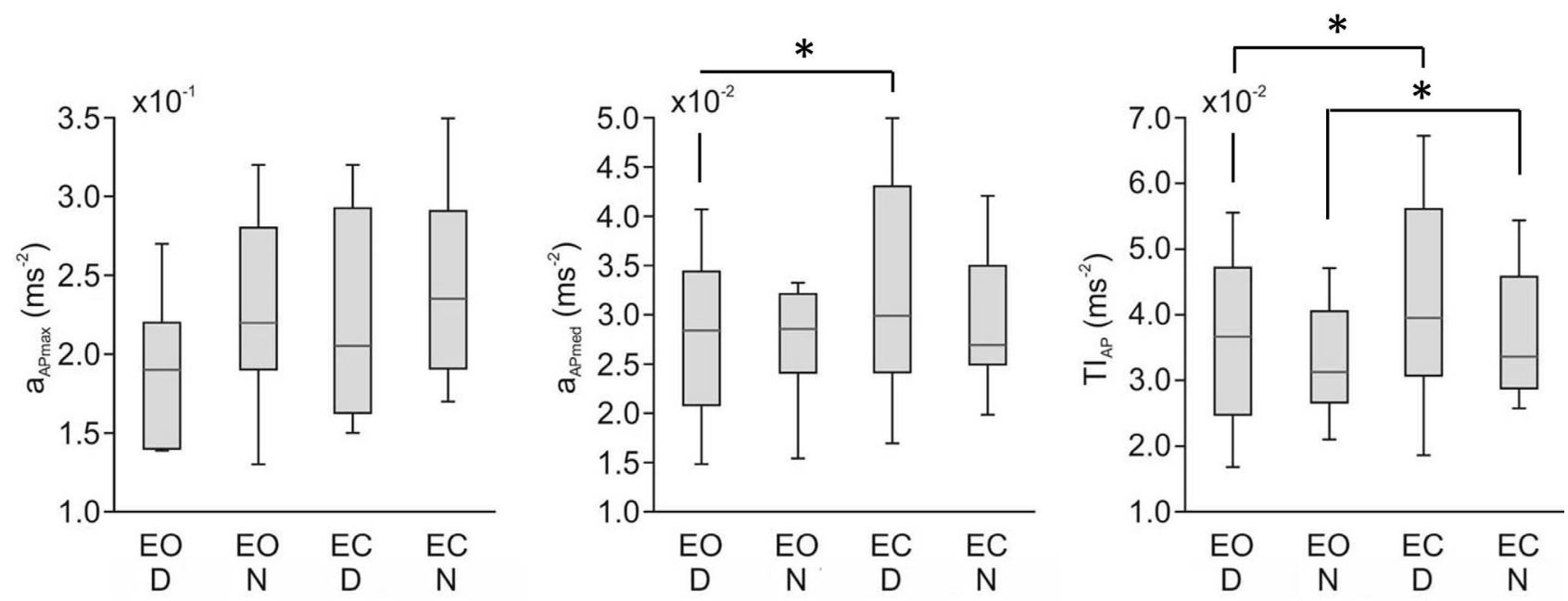

Figure 4. Comparison of maximum (max) and median (med) anterior-posterior accelerations and tremor intensity (TI) of anterior-posterior acceleration of dominant (D) and non-dominant (N) upper arms of subjects standing with eyes open (EO) and eyes closed (EC). *statistically significant difference.
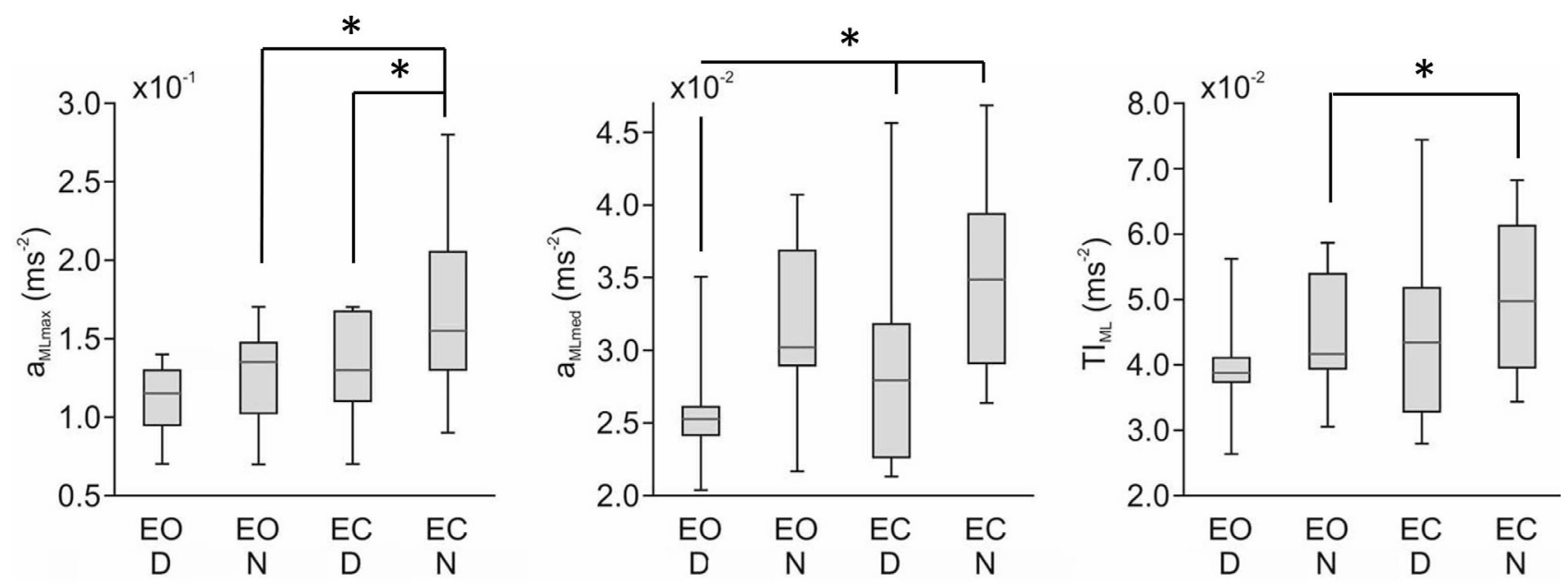

Figure 5. Comparison of maximum (max) and median (med) medio-lateral accelerations and tremor intensity (TI) of medio-lateral acceleration of dominant (D) and non-dominant (N) upper arms of subjects standing with eyes open (EO) and eyes closed (EC). *statistically significant difference.

during stand with EO or EC found significant differences when measuring non-dominant arms of subjects standing with EC, see Table 1.

The comparison of values of tremor intensity of the dominant and non-dominant arm did not find significant differences between the data. In all cases, the comparison of values of tremor intensities of the same arm measured during standing with EO or EC found significant differences, see Table 1.

In all cases of the comparison of the groups of data, the effect sizes were moderate to large, i.e. calculated values were greater than 0.4 in all cases. The differences between groups of data were identified as statistically significant.

\section{Correlation between values on the dominant and non- dominant arms}

In all cases of the comparison of values of maximal accelerations of the dominant and non-dominant arm, the Spearman's rank correlation coefficient indicates a strong correlation (a value is considered as strong if greater than .60 ), i.e. strong positive relationship, between the movements of the dominant and nondominant arm, see Table 2.

In the cases of the comparison of values of median accelerations of the dominant and non-dominant arm in SI direction, the Spearman's rank correlation coefficient indicates strong correlation. Medians of accelerations of the dominant and non-dominant arm in 
Table 1

The calculated p-values from the Wilcoxon test and Cohen's $d$ to assess the differences between the results of measurements of dominant and non-dominant arm, and measurements with $E O$ and $E C$

\begin{tabular}{|c|c|c|c|c|c|c|}
\hline & \multicolumn{2}{|c|}{ SI } & \multicolumn{2}{|c|}{$\mathrm{AP}$} & \multicolumn{2}{|c|}{ ML } \\
\hline & $p$ & $d$ & $p$ & $d$ & $p$ & $d$ \\
\hline \multicolumn{7}{|l|}{$a_{\max }$} \\
\hline EO D vs. EO N & .19 & 0.4 & .19 & 0.7 & .10 & 0.5 \\
\hline EC D vs. EC N & $.01 *$ & 1.1 & .20 & 0.6 & $.02 *$ & 0.8 \\
\hline EO D vs. EC D & $>.99$ & 0.1 & .23 & 0.6 & .07 & 0.6 \\
\hline EO N vs. EC N & .07 & 0.7 & .41 & 0.2 & $.05^{*}$ & 0.9 \\
\hline \multicolumn{7}{|l|}{$a_{\text {med }}$} \\
\hline EO D vs. EO N & .70 & 0.1 & .68 & 0.1 & $.01^{*}$ & 1.2 \\
\hline EC D vs. EC N & .85 & 0.1 & .19 & 0.7 & $.05^{*}$ & 0.8 \\
\hline EO D vs. EC D & $.03 *$ & 0.5 & $.02 *$ & 0.5 & $.04^{*}$ & 0.6 \\
\hline EO N vs. EC N & $.02 *$ & 0.6 & .19 & 0.4 & $.03^{*}$ & 0.5 \\
\hline \multicolumn{7}{|l|}{ TI } \\
\hline EO D vs. EO N & .77 & 0.2 & .38 & 0.3 & .11 & 0.6 \\
\hline EC D vs. EC N & .63 & 0.1 & .19 & 0.4 & .23 & 0.4 \\
\hline EO D vs. EC D & $.02 *$ & 0.4 & $.01^{*}$ & 0.5 & .06 & 0.6 \\
\hline EO N vs. EC N & $.01 *$ & 0.7 & $.02 *$ & 0.4 & $.01^{*}$ & 0.6 \\
\hline
\end{tabular}

Note. $\mathrm{SI}=$ superior-inferior direction; $\mathrm{AP}=$ anterior-posterior direction; $\mathrm{ML}=$ medio-lateral direction; $\mathrm{D}=$ dominant upper $\operatorname{arm} ; \mathrm{N}=$ non-dominant upper $\operatorname{arm} ; \mathrm{EO}=$ eyes open; $\mathrm{EC}=$ eyes closed. *significant difference.

ML direction have a weak or moderate correlation. In the case of the median accelerations in AP direction, the stronger correlation is evident only when subjects standing with EO, see Table 2.

In the cases of the comparison of values of tremor intensity of the dominant and non-dominant arm in SI and AP direction, the Spearman's rank correlation coefficient indicates a strong correlation. Tremor intensities of the dominant and non-dominant arm in ML direction have strong or moderate correlation, see Table 2.

\section{Correlation between the superior-inferior, medio-lateral} and anterior-posterior accelerations

In almost all cases of the comparison of values of maximal accelerations of the dominant upper arm in the SI, $\mathrm{AP}$ and ML direction, the Spearman's rank correlation coefficient indicates a moderate or strong correlation, i.e. positive relationship, between the three accelerations, see Table 3. In only two cases of the comparison of values of maximal accelerations of the non-dominant upper arm in the SI, AP and ML direction, the Spearman's rank correlation coefficient do not indicate significant correlation between the accelerations in the SI, AP or ML direction.
In almost all cases of the comparison of values of median accelerations of the dominant upper arm in the SI, AP and ML direction, the Spearman's rank correlation coefficient indicates a significant correlation between the three accelerations, see Table 3. Also, in only two cases of the comparison of values of median

Table 2

Spearman's rank correlation coefficients between the data measured on dominant and on non-dominant upper limb

\begin{tabular}{cccc}
\hline & SI & AP & ML \\
\hline $\mathrm{a}_{\text {max }}$ & & & \\
EO D vs. EO N & $.62^{*}$ & $.68^{*}$ & $.72^{*}$ \\
EC D vs. EC N & $.67^{*}$ & $.74^{*}$ & $.65^{*}$ \\
$\mathrm{a}_{\text {med }}$ & & & \\
EO D vs. EO N & $.69^{*}$ & .47 & .23 \\
EC D vs. EC N & $.85^{* *}$ & $.79^{*}$ & .47 \\
TI & & & \\
EO D vs. EO N & $.79^{*}$ & $.83^{* *}$ & .52 \\
EC D vs. EC N & $.81^{* *}$ & $.71^{*}$ & $.71^{*}$ \\
\hline
\end{tabular}

Note. $\mathrm{SI}=$ superior-inferior direction; $\mathrm{AP}=$ anterior-posterior direction; $\mathrm{ML}=$ medio-lateral direction; $\mathrm{D}=$ dominant upper arm; $\mathrm{N}=$ non-dominant upper arm; EO = eyes open; $\mathrm{EC}=$ eyes closed. *strong correlation $(r>.60),{ }^{* *}$ very strong correlation $(r>.80)$. 
Table 3

Spearman's rank correlation coefficients between the superior-inferior, medio-lateral and anterior-posterior accelerations

\begin{tabular}{lllll}
\hline & EO D & EO N & EC D & EC N \\
\hline $\mathrm{a}_{\text {max }}$ & & & & \\
SI vs. AP & .49 & .15 & $.79^{*}$ & .45 \\
AP vs. ML & $.67 *$ & $.64^{*}$ & $.66^{*}$ & .43 \\
ML vs. SI & $.75^{*}$ & .27 & $.77^{*}$ & $.79^{*}$ \\
$\mathrm{a}_{\text {med }}$ & & & & \\
SI vs. AP & $.93^{* *}$ & .30 & $.84^{* *}$ & $.60^{*}$ \\
AP vs. ML & .47 & .34 & $.79^{*}$ & .47 \\
ML vs. SI & .36 & $.74^{*}$ & $.76^{*}$ & $.64^{*}$ \\
TI & & & & \\
SI vs. AP & $.93^{* *}$ & $.60^{*}$ & $.81^{* *}$ & $.62^{*}$ \\
AP vs. ML & $.75^{*}$ & .41 & $.88^{* *}$ & .46 \\
ML vs. SI & $.81^{* *}$ & $.71 *$ & $.75^{*}$ & $.72^{*}$ \\
\hline
\end{tabular}

Note. $\mathrm{EO}=$ eyes open; $\mathrm{EC}=$ eyes closed $; \mathrm{SI}=$ superior-inferior direction; $\mathrm{AP}=$ anterior-posterior direction; $\mathrm{ML}=$ medio-lateral direction; $\mathrm{D}=$ dominant upper arm; $\mathrm{N}=$ non-dominant upper arm. *strong correlation $(r>.60),{ }^{* *}$ very strong correlation $(r>.80)$.

accelerations of the non-dominant upper arm in the SI, $\mathrm{AP}$ and ML direction, the Spearman's rank correlation coefficient does not indicate any moderate or strong correlation between the three accelerations.

In all cases of the comparison of values of tremor intensity of the dominant upper arm in the SI, AP and ML direction, the Spearman's rank correlation coefficient indicates a significant correlation between the three tremor intensities, see Table 3. Also, in all cases of the comparison of values of tremor intensity of the non-dominant upper arm in the SI, AP and ML direction, the Spearman's rank correlation coefficient indicates significant correlation between the three tremor intensities.

\section{Discussion}

This study proposed and tested the application of IMUs for measuring spontaneous arm movements while performing a quiet stance. Significant differences in movements of dominant and non-dominant limbs were found using accelerometers in all anatomical axes. Tremors can have generally two sources - oscillations driven by mechanical properties of the limb in the corresponding resonance frequency and central oscillatory component, which synchronizes the motor units' activity of muscles responsible for the segment movements. Several studies have documented that the
PT in the upper extremity is largely driven by the limb mechanics enhanced by stretch reflex oscillation, but with minimal central influences (Elble, 2003; Raethjen et al., 2004; Raethjen, Pawlas, Lindemann, Wenzelburger, \& Deuschl, 2000). In line with this, Carignan, Daneault, and Duval (2012) have found that tremors of all segments are highly correlated and driven by the angular displacement in the shoulder joint.

There are only few data available about spontaneous movements of arms at rest, our results thus represent a pilot set, using modern technique, for further studies (Marsden, 1984). High correlation of tremor intensity between both arms is well documented feature of PT, it shows its common source of oscillation in both extremities. On the contrary, for pathological tremors is typical poor correlation consistent with several central oscillators working in separate loops for each extremity (O'Suilleabhain \& Matsumoto, 1998).

The main applicable findings of this study include significant differences of median accelerations (mediolateral plane) between the dominant and non-dominant upper extremities and between accelerations measured with closed versus open eyes. Less apparent were the differences between the dominant and non-dominant upper extremity and between values measured with closed versus open eyes when parameters of tremor intensity and maximal accelerations were considered, see Table 1. Higher acceleration and higher tremor intensity were recorded on the non-dominant extremity and with closed eyes compared to the open eyes condition. According to the study of Raethjen et al. (2000), side differences in the movements were not age, gender and laterality dependent, but the differences were explained by the segment weight and dimensions (i.e. sex differences are indirect consequence of weight alteration). Effects of laterality, observed in our subjects, could be explained by differences in arm weight between dominant and non-dominant extremity as mentioned in Raethjen et al. (2000). Side differences between dominant versus non-dominant arm weight can amount up to about $4.8 \%$ (Clauser, McConville, \& Young, 1969). Side differences in the grip and elbow strength due to differences in muscle mass were found by Aoki and Demura (2008). Thus, a possible explanation could be that a lighter, non-dominant extremity trembles more. Only a few notions on the tremor correlation with upper limb laterality are found in the literature, e.g. Machowska-Majchrzak et al. (2011) stated that the essential tremor was more intense in the non-dominant extremity in $62 \%$ of patients. On the other hand, no correlation of upper limb movement in walking with laterality was found (Kuhtz-Buschbeck, Brockmann, Gilster, Koch, \& Stolze, 2008). 
The statistically significant difference of the tremor intensity between open and closed eyes conditions is difficult to explain and no relevant literature citations are known. One possible explanation could be that it takes more effort to attain proper posture in space without visual control. For this reason, muscle activity increases and central oscillations are added to the pure mechanical oscillations of the undisturbed stand. This hypothesis could be easily tested by EMG recording of the trembling segments in the future. However, in the case of using accelerometers, the significant difference proved the importance and necessity to take the type of stance task into account, since the results differed between tested conditions. This concludes that complicating stance task by reducing the visual perception, enhances the differences in arm movements.

Methods based on the calculation of the median and finding the maxima of measured values of accelerations are easy to interpret for medical personnel, and therefore mentioned indicators were used. These methods are commonly used for the evaluation of center of pressure (CoP) movement during quiet standing, and therefore the parameters are also used for the evaluation of movement of arms during quiet standing. Median and maxima of measured values are parameters of time-domain analysis. As a method of frequency-domain analysis, tremor intensity was chosen for the assessment of the movement of the upper limbs. The method was chosen as a promising one and because this parameter has not been used before for evaluating the movement of upper limbs during quiet standing. Therefore application of the parameter is an original evaluation procedure of upper limb movement.

There are potential limitations to our study. The main weakness of this study is a small number of subjects, which makes small numbers error possible. Clearly, this study needs to be replicated using a larger sample. Furthermore, measurement during postural load and on several segments of the upper extremity should be included in the future experiments.

However, to test the basic attributes of the application of IMU and quantitative methods proposed for the study of spontaneous arm movements in this preliminary study, a sample of fourteen subjects seems sufficient, just as it was in similar works focused on the spontaneous movements evaluation published by Diener et al. (1992).

\section{Conclusions}

The results pointed to significant differences between movements of the dominant and non-dominant arms during quiet stance with eyes open and eyes closed in a few cases. The results set up pilot values for spontaneous arm movements in rest, without postural load in a group of healthy controls. The designed methods and medical findings may facilitate a wider use of IMUs and evaluation of spontaneous upper extremity movements in medical practice in the future.

\section{Acknowledgment}

This work was done in the framework of research project SGS16/109/OHK4/1T/17 of CTU in Prague. The authors would also like to thank Pavel Smrcka, Karel Hana, Vladimir Socha and Stanislav Kusmirek for the supporting our research and preparation of format of our manuscript.

\section{Conflict of interest}

There were no conflicts of interest.

\section{References}

Allum, J. H., Nijhuis, L. B. O., \& Carpenter, M. G. (2008). Differences in coding provided by proprioceptive and vestibular sensory signals may contribute to lateral instability in vestibular loss subjects. Experimental Brain Research, 184, 391-410.

Altun, K., \& Barshan, B. (2012). Pedestrian dead reckoning employing simultaneous activity recognition cues. Measurement Science and Technology, 23, 025103.

Aoki, H., \& Demura, S. (2008). Characteristics and lateral dominance of hand grip and elbow flexion powers in young male adults. Journal of Physiological Anthropology, 27, 201-206.

Aw, S. T., Halmagyi, G. M., Black, R. A., Curthoys, I. S., Yavor, R. A., \& Todd, M. J. (1998). Head impulses reveal loss of individual semicircular canal function. Journal of Vestibular Research: Equilibrium \& Orientation, 9, 173-180.

Brinckmann, P., Frobin, W., \& Leivseth, G. (2002). Musculoskeletal biomechanics. Stuttgart, Germany: Georg Thieme Verlag.

Carignan, B., Daneault, J. F., \& Duval, C. (2012). The organization of upper limb physiological tremor. European Journal of Applied Physiology, 112, 1269-1284.

Clauser, C. E., McConville, J. T., \& Young, J. W. (1969). Weight, volume, and center of mass of segments of the human body [AMRL Technical Report]. Wright-Patterson Air Force Base, OH: Air Force Systems Command, Aerospace Medical Division.

Cohen, J. (1988). Statistical power analysis for the behavioral sciences. Hillsdale, NJ: Lawrence Erlbaum.

Coolican, H. (1990). Research methods and statistics in psychology. London, United Kingdom: Hodder \& Stoughton Educational. 
Diener, H. C., Dichgans, J., Guschlbauer, B., Bacher, M., Rapp, H., \& Klockgether, T. (1992). The coordination of posture and voluntary movement in patients with cerebellar dysfunction. Movement Disorders, 7, 14-22.

Duclos, C., Nadeau, S., \& Lecours, J. (2008). Lateral trunk displacement and stability during sit-to-stand transfer in relation to foot placement in patients with hemiparesis. Neurorehabilitation and Neural Repair, 22, 715-722.

Elble, R. J. (2003). Characteristics of physiologic tremor in young and elderly adults. Clinical Neurophysiology, 114, 624-635.

Findling, O., Sellner, J., Meier, N., Allum, J. H., Vibert, D., Lienert, C., \& Mattle, H. P. (2011). Trunk sway in mildly disabled multiple sclerosis patients with and without balance impairment. Experimental Brain Research, 213, 363-370.

Fritz, C. O., Morris, P. E., \& Richler, J. J. (2012). Effect size estimates: Current use, calculations, and interpretation. Journal of Experimental Psychology: General, 141, 2.

Gil-Agudo, Á., de Los Reyes-Guzman, A., Dimbwadyo-Terrer, I., Peñasco-Martín, B., Bernal-Sahún, A., López-Monteagudo, P., ... Pons, J. L. (2013). A novel motion tracking system for evaluation of functional rehabilitation of the upper limbs. Neural Regeneration Research, 8, 1773-1782.

Hogg, R. V., \& Craig, A. T. (1995). Introduction to mathematical statistics (5th ed.). New York, NY: Macmillan.

Honegger, F., Van Spijker, G. J., \& Allum, J. H. J. (2012). Coordination of the head with respect to the trunk and pelvis in the roll and pitch planes during quiet stance. Neuroscience, 213, 62-71.

Kennie, T. J., \& Petrie, G. (2010). Engineering surveying technology. Boca Raton, FL: CRC Press.

Khasnis, A., \& Gokula, R. M. (2003). Romberg's test. Journal of Postgraduate Medicine, 49, 169-172.

Konrad, P. (2005). The ABC of EMG. A practical introduction to kinesiological electromyography. Scottsdale, AZ: Noraxon, Inc.

Kuhtz-Buschbeck, J. P., Brockmann, K., Gilster, R., Koch, A., \& Stolze, H. (2008). Asymmetry of arm-swing not related to handedness. Gait \& Posture, 27, 447-454.

Kutílek, P., Socha, V., Čakrt, O., Schlenker, J., \& Bizovská, L. (2015). Trajectory length of pitch vs. roll: Technique for assessment of postural stability. Acta Gymnica, 45, 85-92.

Louis, E. D. (2005). Essential tremor. Lancet Neurology, 4, $100-110$.

Liu, J., Zhang, X., \& Lockhart, T. E. (2012). Fall risk assessments based on postural and dynamic stability using inertial measurement unit. Safety and Health at Work, 3, 192-198.

Machowska-Majchrzak, A., Pierzchała, K., Pietraszek, S., \& Łabuz-Roszak, B. (2011). Essential tremor-assessment of tremor accelerometric parameters' symmetry and the relationship between hand dominance and severity of tremor. Neurologia i Neurochirurgia Polska, 45, 121-127.

Machowska-Majchrzak, A., Pierzchała, K., Pietraszek, S., Łabuz-Roszak, B., \& Bartman, W. (2012). The usefulness of accelerometric registration with assessment of tremor parameters and their symmetry in differential diagnosis of parkinsonian, essential and cerebellar tremor. Neurologia $i$ Neurochirurgia Polska, 46, 145-156.

Mancini, M., Salarian, A., Carlson-Kuhta, P., Zampieri, C., King, L., Chiari, L., \& Horak, F. B. (2012). ISway: A sensitive, valid and reliable measure of postural control. Journal of NeuroEngineering and Rehabilitation, 9, 59.

Marsden, C. D. (1984). Origins of normal and pathological tremor. In L. J. Findley \& R. Capildeo (Eds.), Movement disorders: Tremor (pp. 37-84). London, United Kingdom: Palgrave Macmillan UK.

Melecky, R., Socha, V., Kutilek, P., Hanakova, L., Takac, P., Schlenker, J., \& Svoboda, Z. (2016). Quantification of trunk postural stability using convex polyhedron of the time-series accelerometer data. Journal of Healthcare Engineering, 2016, 1621562.

O'Suilleabhain, P. E., \& Matsumoto, J. Y. (1998). Time-frequency analysis of tremors. Brain, 121, 2127-2134.

Oldfield, R. C. (1971). The assessment and analysis of handedness: The Edinburgh inventory. Neuropsychologia, 9, 97-113.

Osler, C. J., \& Reynolds, R. F. (2012). Postural reorientation does not cause the locomotor after-effect following rotary locomotion. Experimental Brain Research, 220, 231-237.

Papapetropoulos, S., Katzen, H. L., Scanlon, B. K., Guevara, A., Singer, C., \& Levin, B. E. (2010). Objective quantification of neuromotor symptoms in Parkinson's disease: Implementation of a portable, computerized measurement tool. Parkinson's Disease, 2010, 760196.

Rodríguez, R., Costa, U., Torrent, M., Solana, J., Opisso, E., Cáceres, C., ... Gómez, E. J. (2010). Upper limb portable motion analysis system based on inertial technology for neurorehabilitation purposes. Sensors, 10, 10733-10751.

Raethjen, J., Lauk, M., Köster, B., Fietzek, U., Friege, L., Timmer, J., ... Deuschl, G. (2004). Tremor analysis in two normal cohorts. Clinical Neurophysiology, 115, 2151-2156.

Raethjen, J., Pawlas, F., Lindemann, M., Wenzelburger, R., \& Deuschl, G. (2000). Determinants of physiologic tremor in a large normal population. Clinical Neurophysiology, 111, 1825-1837.

Schubert, P., Kirchner, M., Schmidtbleicher, D., \& Haas, C. T. (2012). About the structure of posturography: Sampling duration, parametrization, focus of attention (Part I). Journal of Biomedical Science and Engineering, 5, 496-507.

Stirling, D. A., Hesami, A. S., Ritz, C. H., Adistambha, K., \& Naghdy, F. (2010). Symbolic modelling of dynamic human motions. Rijeka, Croatia: InTech.

Ying, N., \& Kim, W. (2002). Use of dual Euler angles to quantify the three-dimensional joint motion and its application to the ankle joint complex. Journal of Biomechanics, 35, 1647-1657.

Zadnikar, M., \& Rugelj, D. (2011). Postural stability after hippotherapy in an adolescent with cerebral palsy. Journal of Novel Physiotherapies, 1, 106. 